\title{
Multiscale optical imaging of rare- earth-doped nanocomposites in a small animal model
}

Laura M. Higgins

Vidya Ganapathy

Harini Kantamneni

Xinyu Zhao

Yang Sheng

Mei-Chee Tan

Charles M. Roth

Richard E. Riman

Prabhas V. Moghe

Mark C. Pierce 


\section{Multiscale optical imaging of rare-earth- doped nanocomposites in a small animal model}

\author{
Laura M. Higgins, ${ }^{\text {a }}$ Vidya Ganapathy, ${ }^{\text {a }}$ \\ Harini Kantamneni, ${ }^{b}$ Xinyu Zhao, ${ }^{c}$ Yang Sheng, ${ }^{c}$ \\ Mei-Chee Tan, ${ }^{c}$ Charles M. Roth, ${ }^{a, b}$ Richard E. Riman, ${ }^{d}$ \\ Prabhas V. Moghe, ${ }^{a, b}$ and Mark C. Pierce ${ }^{a, *}$ \\ ${ }^{a}$ Rutgers, The State University of New Jersey, Department of \\ Biomedical Engineering, Piscataway, New Jersey, United States \\ ${ }^{\mathrm{b}}$ Rutgers, The State University of New Jersey, Department of \\ Chemical and Biochemical Engineering, Piscataway, New Jersey, \\ United States \\ 'Singapore University of Technology and Design, Engineering \\ Product Development, Singapore \\ ${ }^{\mathrm{d}}$ Rutgers, The State University of New Jersey, Department of \\ Materials Science and Engineering, Piscataway, New Jersey, \\ United States
}

\begin{abstract}
Rare-earth-doped nanocomposites have appealing optical properties for use as biomedical contrast agents, but few systems exist for imaging these materials. We describe the design and characterization of (i) a preclinical system for whole animal in vivo imaging and (ii) an integrated optical coherence tomography/confocal microscopy system for high-resolution imaging of ex vivo tissues. We demonstrate these systems by administering erbiumdoped nanocomposites to a murine model of metastatic breast cancer. Short-wave infrared emissions were detected in vivo and in whole organ imaging ex vivo. Visible upconversion emissions and tissue autofluorescence were imaged in biopsy specimens, alongside optical coherence tomography imaging of tissue microstructure. We anticipate that this work will provide guidance for researchers seeking to image these nanomaterials across a wide range of biological models. @ 2018 Society of Photo-Optical Instrumentation Engineers (SPIE) [DOI: 10.1117/1.JBO.23.3.030505]
\end{abstract}

Keywords: short-wave infrared imaging; NIR-II imaging; confocal microscopy; preclinical imaging; multimodal imaging.

Paper 170624LRR received Sep. 28, 2017; accepted for publication Feb. 26, 2018; published online Mar. 21, 2018.

\section{Introduction}

There is growing interest in the development of luminescent optical probes emitting at wavelengths beyond $1000 \mathrm{~nm}$, in the shortwave infrared (SWIR) or NIR-II spectral region. ${ }^{1-4}$ These probes aim to take advantage of low scattering, low autofluorescence, and minima in the absorption spectra of water, lipid, and collagen to enable optical imaging at increased depths in tissue, compared with visible and NIR-I emitting fluorophores. ${ }^{5-7}$ We have been investigating rare-earth-doped nanomaterials, which simultaneously provide both SWIR and visible emissions under continuous wave near-infrared $(980 \mathrm{~nm})$ excitation. $^{7}$ Although rare-earth-based probe development has gained significant

*Address all correspondence to: Mark C. Pierce, E-mail: mark.pierce@rutgers .edu traction in recent years, ${ }^{1-8}$ there is currently a lack of platforms available for imaging these nanomaterials across the whole animal to subcellular scales.

There are several commercial small animal imaging platforms designed for imaging fluorescent and bioluminescent probes, which emit in the visible and NIR-I regions. To the best of our knowledge, there are no commercial small animal whole-body imagers that can work with probes that emit at longer wavelengths, such as the rare-earth-doped nanomaterials used here. For high-resolution imaging, commercial epifluorescence microscopes are typically configured for excitation with UV/visible wavelengths and imaging of longer wavelength emissions. Upconversion light from rare-earth nanocomposites is at shorter wavelengths than the near-infrared excitation. Also, point-scanning confocal or multiphoton microscopes are poorly suited to imaging rare-earth probes because of the unusually long emission lifetime of these materials (milliseconds) compared with conventional fluorescent dyes (nanoseconds). The short pixel dwell time of these scanning microscopes (on the order of $100 \mathrm{~ns}$ ) results in very little of the rare-earth emission being collected due to its long emission lifetime.

To overcome these limitations, we describe the design, assembly, and characterization of two platforms for rare-earthbased nanoprobe imaging. The first is a preclinical in vivo imaging system using SWIR emissions for deep tissue imaging. The second is an integrated optical coherence tomography (OCT) and multichannel line-scanning confocal microscopy system for high-resolution imaging of ex vivo tissues. This system uses backscattered SWIR light for OCT and visible upconversion emissions for confocal imaging. We demonstrate the capabilities of these systems by imaging rare-earth-doped nanoprobes administered to a murine model of metastatic breast cancer.

\section{Methods}

\subsection{Wide-Field Short-Wave Infrared Imaging System}

A fully automated system was developed for in vivo SWIR imaging in small animals [Figs. 1(a) and 1(b)]. Compared with our previous generation system, ${ }^{4}$ the spatial resolution of the system reported here is $4 \times$ greater, animal imaging is performed by automated scanning of the illumination beam, the emission filter combination has over $3 \times$ higher transmission, and real-time overlay of rare-earth signal has been implemented. The distal tip of a multimode fiber-coupled 980-nm diode laser (Opto Engine LLC) was mounted on a dual-axis motorized translation stage (Zaber, X-LSM-E) and the output beam collimated to a $9.6 \mathrm{~mm}\left(1 / e^{2}\right)$ diameter (Thorlabs, F810SMA-780). An InGaAs camera with $512 \times 640$ pixels, $25-\mu$ m square, (Sensors Unlimited, 640HSX) was fitted with a 25-mm fixed focal length lens (StingRay Optics, SR0907, $f / 1.4$ ), providing a $195 \mathrm{~mm} \times$ $155 \mathrm{~mm}$ field-of-view at a 300-mm working distance [Fig. 1(c)]. The translation stage raster scans the illumination beam across the sample platform while the camera continuously transfers images to a CameraLink frame grabber (NI PCIe-1427) at 30 frames per second. A custom LabVIEW VI creates an image from SWIR emissions by retaining the maximum intensity value at each pixel as images are acquired. After the scan is complete, a 1550-nm LED (Thorlabs, LED1550L) is turned on and a single full-field reflectance image is acquired for coregistration

\footnotetext{
$1083-3668 / 2018 / \$ 25.00$ @ 2018 SPIE
} 


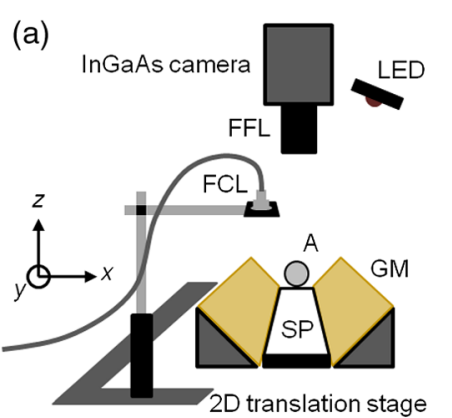

(c)

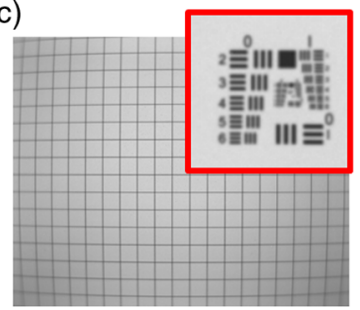

(d) 250
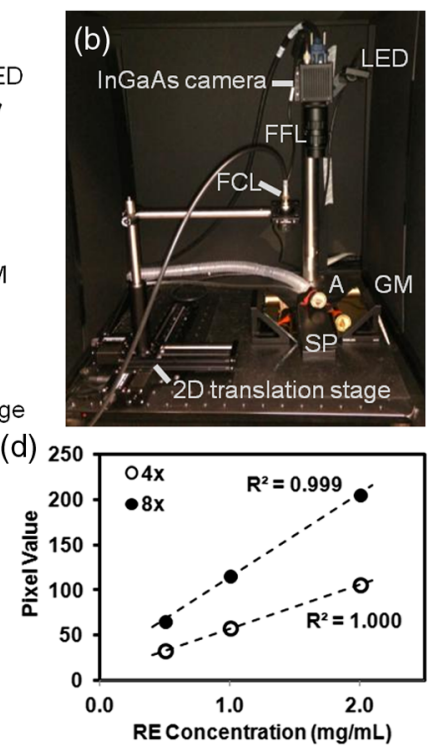

Fig. 1 Preclinical SWIR imaging platform. (a) Schematic diagram; SP: sample platform, FCL: fiber-coupled laser, FFL: fixed focal length lens, A: anesthesia, SP: sample pad, GM: gold mirrors. (b) Photograph of the SWIR imager. (c) Full field-of-view (1-cm grid) and USAF target (inset). (d) Measured response of the imaging platform to rare-earth-doped phantoms at $4 \times$ and $8 \times$ camera digital gain.

and overlay of SWIR emissions with anatomical features. Two gold mirrors are mounted at \pm 45 deg on either side of the sample platform for simultaneous imaging of left and right lateral views. For imaging animals receiving erbium-doped nanoparticles (SWIR emission peak at $1550 \mathrm{~nm}$ ), a stack of two 1350-nm long-pass filters (Thorlabs) and one 1497- to 1579-nm bandpass filter (Semrock) was mounted directly onto the camera lens.

A 1951 USAF target was imaged under 1550-nm illumination, demonstrating the ability to resolve $0.5-\mathrm{mm}$ features (group 0, element 1) at $31 \%$ contrast [Fig. 1(c)]. Silicone phantoms containing $0.5 \times 10^{-3}, 1 \times 10^{-3}$, and $2 \times 10^{-3}$ erbiumdoped nanoparticles $/ \mathrm{mL}$ were placed on the sample platform and imaged to assess the degree of linearity between nanoparticle concentration and measured signal intensity [Fig. 1(d)].

\subsection{Optical Coherence Tomography/Confocal Microscopy Imaging System}

For high-resolution imaging, the sample arm of a spectral-domain OCT system was integrated within a dual-channel line-scanning confocal microscope (Fig. 2). Line scanning allows imaging with pixel exposure times far longer than point-scanning systems, which is important when imaging rare-earth-doped nanoparticles due to their long emission lifetimes. ${ }^{9}$ The OCT source (Thorlabs, SLD1325, $\lambda_{0}=1325 \mathrm{~nm}, \Delta \lambda_{\mathrm{FWHM}}=100 \mathrm{~nm}$ ) is collimated to a 3.4-mm diameter beam $\left(1 / e^{2}\right)$ using a 19-mm focal length achromatic doublet lens (Thorlabs, L3). Confocal illumination light from a 980-nm single-mode fiber-coupled laser (QPhotonics, QFBGLD-980-500) is collimated to an 8.0-mm diameter by a 36.6-mm focal length module (Thorlabs, L1). A 150-mm focal length achromatic cylindrical lens (Thorlabs, L2) forms a focused illumination line at the back aperture of the objective lens, which is positioned as close as possible to the galvo-mounted mirror. A dichroic mirror (Semrock, FF495-Di03, D1), with a reflection band at the OCT source wavelength combines the illumination beams along a coaxial path. Both beams are then reflected by an 805-nm short-pass dichroic mirror (Thorlabs, DMSP805,

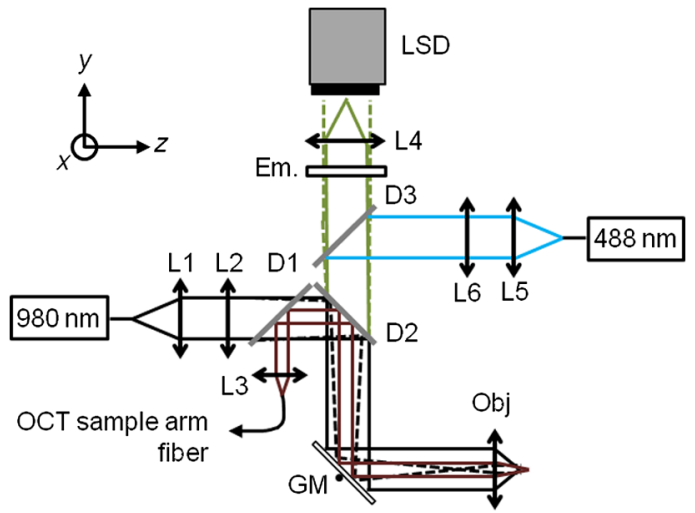

Fig. 2 Integrated OCT/line-scanning confocal imaging system. L1-6: lenses/lens assemblies, D1-3: dichroic mirrors, GM: galvo-mounted mirror, Obj: objective lens, Em: emission filter, LSD: line-scanning detector.

D2) used to separate the nanoparticle excitation and (upconversion) emission wavelengths. To additionally allow for tissue autofluorescence excitation, a single-mode fiber-coupled laser with 488-nm wavelength (Blue Sky Research, FiberTec II) was collimated to an $8.0-\mathrm{mm}$ diameter using a $35-\mathrm{mm}$ focal length aspherized achromatic lens (Edmund Optics, L5) and then reshaped by a $150-\mathrm{mm}$ focal length achromatic cylindrical lens (Thorlabs, L6). This beam is reflected by a 495-nm long-pass dichroic mirror (Semrock, D3), which transmits autofluorescence and rare-earth upconversion emissions. All three illumination beams are reflected at the galvo mirror. A $20 \times / 0.40$ objective lens (Olympus) was used for confocal imaging, whereas a 40-mm focal length achromatic doublet lens (Thorlabs) was used for OCT. Erbium-doped nanoparticle emissions were transmitted through a 519- to 700-nm band-pass filter (Semrock) and focused by a $200-\mathrm{mm}$ focal length achromatic doublet lens (Thorlabs, L4) onto a line-scan camera (Aviiva, EM1) with a single row of 1024 pixels, each $14-\mu \mathrm{m}$ wide and $28-\mu \mathrm{m}$ tall. The height of the pixels was $\sim 0.76$ Airy units; therefore, no additional confocal slit was used. Backscattered OCT light returned through the sample arm fiber to the detection spectrometer, which was configured with a 1200 l/mm transmissive diffraction grating (Wasatch Photonics) and high-speed InGaAs line scan camera (Sensors Unlimited, LDH-2) as described previously. ${ }^{10}$

A 1951 USAF resolution target was used to measure a confocal lateral resolution of $2.8 \mu \mathrm{m}$. The full width at the half maximum (FWHM) of the mean pixel intensity over the central region of a mirror scanned through the focus indicated an optical sectioning thickness of $11.3 \mu \mathrm{m}$ with the $20 \times / 0.40$ objective. The axial resolution of the OCT system was measured to be $14 \mu \mathrm{m}$ in air over a $2.0-\mathrm{mm}$ imaging depth, by setting a mirror at the focus of the OCT imaging beam and adjusting the reference path length over the imaging range. The OCT system resolved group 5, element 3 on a 1951 USAF resolution target with $34.7 \%$ contrast, corresponding to a lateral resolution of $27.9 \mu \mathrm{m}$. These values can be compared with theoretical figures of $7.7-\mu \mathrm{m}$ axial resolution, 3.0-mm imaging depth, and lateral resolution of $19.8 \mathrm{~mm}$.

\subsection{Rare-Earth-Doped Nanocomposites}

Rare-earth-doped nanocomposites were synthesized using a solvothermal decomposition method, forming a $\mathrm{NaYF}_{4}$ core doped with ytterbium $(\mathrm{Yb})$ and erbium $(\mathrm{Er})$, surrounded by an undoped 
$\mathrm{NaYF}_{4}$ shell. ${ }^{11}$ Under continuous wave excitation at $980 \mathrm{~nm}$, these particles emit fluorescence around $1550 \mathrm{~nm}$, as well as upconversion light around 515, 540, and $650 \mathrm{~nm}$. These nanocomposites $(\sim 10-\mu \mathrm{m}$ diameter $)$ were rendered biocompatible through encapsulation in human serum albumin ${ }^{4}$ and functionalized with the chemokine receptor antagonist AMD3100, forming functionalized rare-earth-doped albumin nanocomposites (fREANCs), each $\sim 100 \mathrm{~nm}$ in diameter and containing $\sim 30$ individual nanoparticles. ${ }^{12}$ AMD3100 has been shown to preferentially bind to cells with high expression levels of CXCR4, a chemokine receptor overexpressed on many types of primary and metastatic cancer. ${ }^{13}$

\subsection{Animal Model}

All animal procedures were carried out under a protocol approved by the Rutgers University Institutional Animal Care and Use Committee. SCP28 cells (provided by Dr. Yibin Kang, Princeton University), derived from the MDA-MB-231 line and transfected with luciferase, were cultured in Dulbecco's modified Eagle's medium supplemented with $10 \%$ fetal bovine serum and $1 \%$ penicillin-streptomycin. Two 5-week-old female athymic nude mice (Taconic Laboratories) were inoculated with 300,000 SCP28 cells via tail vein injection, with two additional animals serving as healthy controls. Tumor uptake and progression were evaluated through weekly bioluminescence imaging (BLI) (Bruker, MS FX PRO). Each animal received an intra-peritoneal injection of D-Luciferin (Perkin Elmer, $150 \mathrm{mg} / \mathrm{kg}$ ). Animals were then anesthetized under inhaled isoflurane at a concentration of $4 \%$. Ten minutes later, animals were transferred to the BLI imaging chamber and imaged under general anesthesia with 1 to $2 \%$ isoflurane. BLI was performed using a 10 -min exposure and $8 \times 8$ pixel binning. For each animal, once tumor uptake was confirmed on BLI, SWIR imaging was performed within $24 \mathrm{~h}$.

\subsection{Small Animal Short-Wave Infrared Imaging Procedures}

For in vivo imaging, animals were anesthetized under $4 \%$ inhaled isoflurane maintained at a concentration of $1 \%$ to $2 \%$. The collimated $980-\mathrm{nm}$ laser beam $[9.6 \mathrm{~mm}$ diameter $\left(1 / e^{2}\right), 1.7 \mathrm{~W}$ power] was continuously scanned over a $100 \mathrm{~mm} \times 50 \mathrm{~mm}$ field at $15 \mathrm{~mm} / \mathrm{s}$. Under these conditions, each individual point on the animal is illuminated for $640 \mathrm{~ms}$ resulting in a delivered fluence of $1.5 \mathrm{~J} / \mathrm{cm}^{2}$, less than half of the maximum permissible exposure under these conditions $\left(3.6 \mathrm{~J} / \mathrm{cm}^{2}\right)$ defined by the American National Standards Institute (ANSI z136.1). The beam has a Gaussian intensity profile rather than a uniform distribution, so some overlap is required between successive sweeps of the raster scan to achieve uniform illumination. A lateral scan step of 2-mm results in 7.6$\mathrm{mm}$ overlap between each sweep and a variation in illumination intensity of $26 \%$. A smaller lateral step would reduce this variation but extend the scan time. Using the above parameters, each scan took $\sim 3$ min to complete. For ex vivo imaging, the lungs and liver from each animal were excised immediately following euthanization. Ex vivo imaging was performed using the same instrument parameters as for in vivo imaging.

Whole animal SWIR images were processed by manually outlining regions-of-interest (ROIs) corresponding to the liver and the lungs in the full-field reflectance images captured pre and post-nanoparticle injection. The mean SWIR pixel value in the pre-injection ROI was subtracted from each of the pixels in the post-injection ROI. These background-corrected pixel values were then displayed as a partially transparent overlay on the post-injection reflectance image with a user-defined colormap. With no pre-injection image of the organs available in the ex vivo cases, a region within the field-of-view that was not illuminated was used to estimate the background level.

\subsection{Optical Coherence Tomography/Confocal Imaging of Biopsy Specimens}

For each of the excised lungs, tissue samples were collected using a 2-mm-diameter punch biopsy, from regions with suspected tumor, and from the lungs of the healthy control animal. After OCT imaging of these biopsies, specimens were frozen at $-80^{\circ} \mathrm{C}$ in optimal cutting temperature compound for cryosectioning. About $60-\mu \mathrm{m}$-thick tissue sections were collected for confocal microscopy and $20-\mu \mathrm{m}$ sections were collected and stained with hematoxylin and eosin (H\&E). The line-scanning confocal microscope was used to examine the specimens and identify areas exhibiting the fREANC upconversion signal. Autofluorescence images of each field were obtained under 488-nm excitation.

\section{Results}

\subsection{Wide-Field Short-Wave Infrared Imaging, In Vivo and Ex Vivo}

Both tumor-bearing animals were imaged in vivo immediately following i.v. administration of fREANCs [Figs. 3(a) and 3(b), " $0 \mathrm{~h}$ " panel]. One animal was euthanized immediately after imaging [Fig. 3(a)] and the other was reimaged $24 \mathrm{~h}$ later [Fig. 3(b), "24 h" panel], then euthanized. SWIR signal was evident in the abdominal region of both animals immediately after fREANC administration. This SWIR signal likely originated from both tumor-bound and free nanocomposites. At $24 \mathrm{~h}$, this abdominal signal was reduced almost to the background

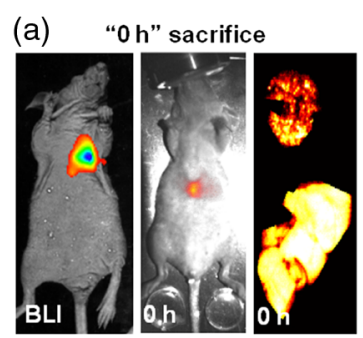

(c) No REANCs

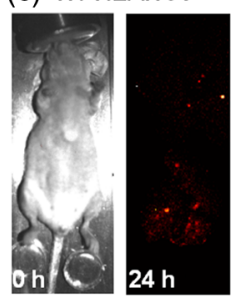

(b)
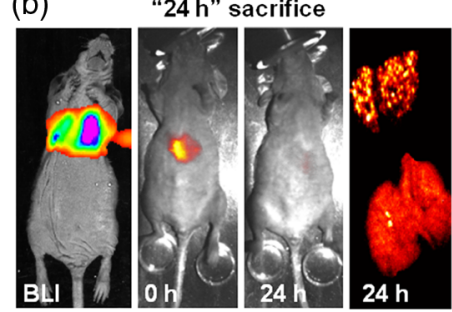

(d)

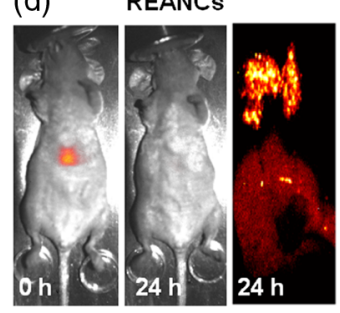

Fig. 3 (a,b) Animals receiving SCP28 breast cancer cells underwent BLI followed by fREANC administration. In vivo SWIR imaging was performed immediately " $0 \mathrm{~h}$ " (a) and at 24-h " 24 h" (b) Ex vivo imaging of the lungs (top organ) and liver (bottom organ) were performed immediately after euthanization. (c,d) Healthy animals were imaged without fREANC administration (c), and immediately post fREANC administration ("O h") and at $24 \mathrm{~h}$ (d). 

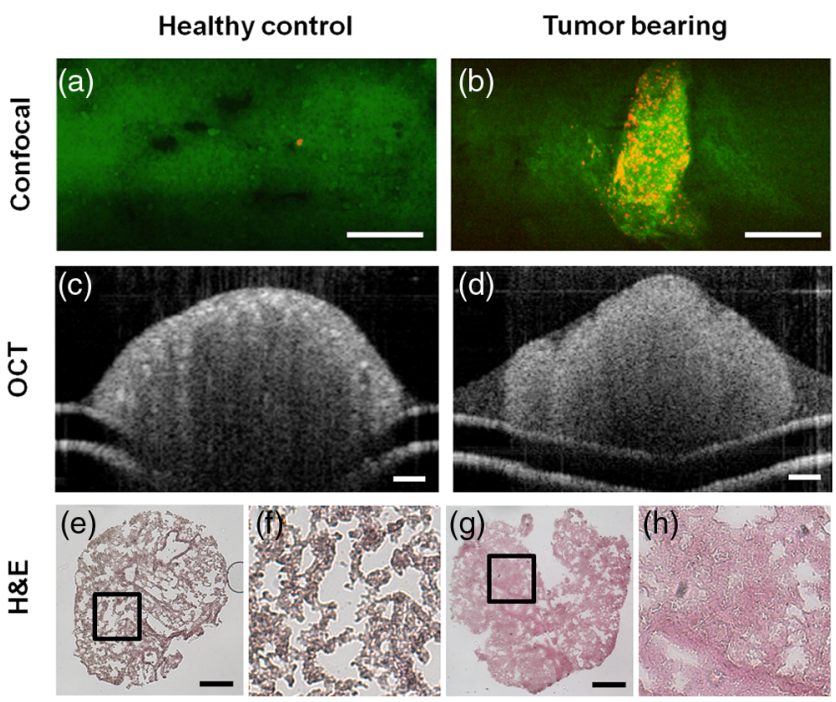

Fig. 4 Confocal microscopy images of nanoparticle upconversion emission (red) overlaid on tissue autofluorescence (green) in (a) tissue specimens from healthy and (b) tumor bearing animals. (c,d) Corresponding OCT images. (e-h) Corresponding H\&E stained histopathology sections. Scale bars $=100 \mu \mathrm{m}$.

level [Fig. 3(b), "24 h" panel], indicating that a substantial proportion of administered nanoparticles cleared the body within this time frame. SWIR images of the excised lungs (top organ) and livers (bottom organ) are shown in the right-hand panels of Figs. 3(a) and 3(b). The liver showed a higher mean signal than the lungs for the animal euthanized immediately following fREANC administration [Fig. 3(a)]. At $24 \mathrm{~h}$, the ex vivo liver [Fig. 3(b)] had lower SWIR intensity than the liver excised immediately after fREANC administration, whereas the lungs at $24 \mathrm{~h}$ exhibited a similar mean SWIR intensity to the "0 h" case.

The healthy control animal that did not receive fREANCs showed no significant SWIR autofluorescence signal in vivo or in organs excised immediately after imaging [Fig. 3(c)]. The control animal that received fREANCs showed abdominal signal on in vivo imaging immediately after fREANC administration [Fig. 3(d), "0 h"], with significantly reduced signal at 24 h. Ex vivo imaging of the lungs and liver of this control animal showed higher mean intensity in the lungs compared with the liver [Fig. 3(d)].

\subsection{High-Resolution Tissue Imaging, Ex Vivo}

Figure 4 shows representative confocal images (a,b) and coregistered OCT images (c,d) of tissue specimens collected at the study end point from the lungs of healthy control $(\mathrm{a}, \mathrm{c})$ and tumor bearing $(b, d)$ animals. The rare-earth nanocomposite up-conversion signal is overlaid (red) on the tissue autofluorescence signal (green). Tissue from the healthy control animals contained confocal fields with a few small discrete regions of fREANC upconversion signal [Fig. 4(a)]. Some tissue sections from the tumor bearing animal had high levels of discrete, localized fREANC up-conversion [e.g., Fig. 4(b)], with other sections appearing similar to healthy tissue, with few discrete regions of fREANC up-conversion. The OCT image from the corresponding normal tissue [Fig. 4(c)] has punctate regions of increased backscattering near the tissue surface, whereas the OCT image of the tumor tissue has a more uniform backscattering signal
[Fig. 4(d)]. On H\&E sections prepared from these specimens, alveolar structure is readily apparent in the healthy tissue [Figs. 4(e) and 4(f)] whereas sections from the tumor exhibit denser tissue with few, small alveoli [Figs. 4(g) and 4(h)].

\section{Conclusions}

This article describes the design and characterization of two imaging systems for evaluating rare-earth-doped nanocomposites in biological systems. Whole animal in vivo imaging and whole organ ex vivo imaging were implemented using scanned excitation light and an InGaAs camera. Ex vivo OCT imaging was integrated with dual-channel confocal microscopy for imaging tissue microstructure, autofluorescence, and nanoparticle distribution. The capabilities of these systems are illustrated by imaging albumin-encapsulated erbium-doped nanocomposites in a mouse model of metastatic breast cancer. We anticipate that this work will guide other researchers studying these emerging nanomaterials in the design and assembly of imaging platforms to suit their specific needs.

\section{Disclosures}

The authors have no relevant financial interests in this article and no potential conflicts of interest to disclose.

\section{Acknowledgments}

This project was supported by the National Institutes of Health, Grant No. R01 EB018378.

\section{References}

1. G. Hong, A. L. Antaris, and H. Dai, "Near-infrared fluorophores for biomedical imaging," Nat. Biomed. Eng. 1, 0010 (2017).

2. O. T. Bruns et al., "Next-generation in vivo optical imaging with shortwave infrared quantum dots," Nat. Biomed. Eng. 1, 0056 (2017).

3. E. Thimsen, B. Sadtler, and M. Y. Berezin, "Shortwave-infrared (SWIR) emitters for biological imaging: a review of challenges and opportunities," Nanophotonics 6(5), 1043-1054 (2017).

4. D. J. Naczynski et al., "Rare-earth-doped biological composites as in vivo shortwave infrared reporters," Nat. Commun. 4, 2199 (2013).

5. R. H. Wilson et al., "Review of short-wave infrared spectroscopy and imaging methods for biological tissue characterization," J. Biomed. Opt. 20(3), 030901 (2015).

6. Q. Cao et al., "Multispectral imaging in the extended near-infrared window based on endogenous chromophores," J. Biomed. Opt. 18(10), 101318 (2013).

7. D. C. Sordillo et al., "Short wavelength infrared optical windows for evaluation of benign and malignant tissues," J. Biomed. Opt. 22(4), 045002 (2017).

8. D. J. Naczynski et al., "Rare earth nanoprobes for functional biomolecular imaging and theranostics," J. Mater. Chem. B 2, 2958-2973 (2014).

9. L. M. Higgins et al., "Line-scanning confocal microscopy for highresolution imaging of upconverting rare-earth-based contrast agents," J. Biomed. Opt. 20(11), 110506 (2015).

10. L. M. Higgins and M. C. Pierce, "Design and characterization of a handheld multimodal imaging device for the assessment of oral epithelial lesions," J. Biomed. Opt. 19(8), 086004 (2014).

11. G.-S. Yi and G.-M. Chow, "Water-soluble $\mathrm{NaYF}_{4}: \mathrm{Yb}, \operatorname{Er}(\mathrm{Tm}) /$ $\mathrm{NaYF}_{4} /$ polymercore/shell/shell nanoparticles with significant enhancement of upconversion fluorescence," Chem. Mater. 19(3), 341-343 (2007).

12. M. Zevon et al., "CXCR-4 targeted, short wave infrared (SWIR) emitting nanoprobes for enhanced deep tissue imaging and micrometastatic cancer lesion detection," Small 11(47), 6347-6357 (2015).

13. M. C. Smith et al., "CXCR4 regulates growth of both primary and metastatic breast cancer," Cancer Res. 64(23), 8604-8612 (2004). 